\title{
Handheld Computing in Medicine
}

Sandra Fischer, MD, Thomas E. Stewart, MD, FRCPC, Sangeeta Mehta, MD, FRCPC, Randy Wax, MD, FRCPC, Stephen E. Lapinsky, MB, BCh, FRCPC

\begin{abstract}
A b s t r a C t Handheld computers have become a valuable and popular tool in various fields of medicine. A systematic review of articles was undertaken to summarize the current literature regarding the use of handheld devices in medicine. A variety of articles were identified, and relevant information for various medical fields was summarized. The literature search covered general information about handheld devices, the use of these devices to access medical literature, electronic pharmacopoeias, patient tracking, medical education, research, business management, e-prescribing, patient confidentiality, and costs as well as specialty-specific uses for personal digital assistants (PDAs).

The authors concluded that only a small number of articles provide evidence-based information about the use of PDAs in medicine. The majority of articles provide descriptive information, which is nevertheless of value. This article aims to increase the awareness among physicians about the potential roles for handheld computers in medicine and to encourage the further evaluation of their use.
\end{abstract}

- J Am Med Inform Assoc. 2003;10:139-149. DOI 10.1197/jamia.M1180.

Since the introduction of handheld computers or personal digital assistants (PDAs) in the early 1990s, they have become increasingly popular for a variety of medical applications. This article reviews the current literature regarding these devices, with an emphasis on identifying the potential roles for PDAs in medicine and the evidence to support their use.

\section{Background}

The prevalent use of PDAs in medicine has stimulated studies of the potential market and role for these devices in clinical medical practice. Analysts predict that by 2004, 20\% of physicians will use handhelds for e-prescribing, ordering and checking lab tests,

Affiliations of the authors: Technology Application Unit, Intensive Care Unit, Mount Sinai Hospital, and Interdepartmental Division of Critical Care, University of Toronto, Toronto, Ontario, Canada.

Correspondence and reprints: Stephen E. Lapinsky, MB, BCh, FRCPC, Director, Technology Application Unit, Associate Director, ICU, Mount Sinai Hospital, 600 University Ave. \#1825, Toronto, Ontario M5G 1X5, Canada; e-mail: <stephen.lapinsky@ utoronto.ca>.

Received for publication: 06/27/02; accepted for publication: $10 / 14 / 02$. capturing charges, and dictating notes. ${ }^{1}$ An early study by Labkoff demonstrated that PDAs clearly had a potential role in medical practice. ${ }^{2}$ A cross-sectional survey of 250 family physicians in 1995 determined that physicians are interested in computerbased information about medications, patient education material, and treatment recommendations. ${ }^{3}$ In this survey, $84.5 \%$ of all physicians and $94.1 \%$ of physicians younger than 40 years of age stated that they would consider carrying a handheld computer. ${ }^{3}$

Currently available PDAs do not have the functionality to manage complete electronic medical records or store large graphics, but they have been identified by users as excellent tools for managing clinical information and accessing it at the point of care. ${ }^{3}$ However, many physicians and information technology professionals are unaware of the variety of potential uses. Although an increasing body of published literature is emerging, it seems to be diffusely spread through a broad spectrum of general and specialty medical journals. This article provides an overview of current literature pertaining to applications of handheld devices in medicine. We believe that such a review is important to increase awareness of this technology and to assist clinicians in making decisions about incorporating PDAs into their practice. 


\section{Methods}

We undertook a systematic review of recent articles to summarize the current literature covering the use of handheld devices in medicine. OVID, a computerized bibliographic record database, was used to identify eligible articles. MEDLINE between 1998 and October 2001 and PREMEDLINE up to June 2002 were used, applying the text words "personal digital assistant," "PDA," "microcomputer," "palm," "handheld," "wireless," and a combination of these search terms. It was noted that several important articles on handheld devices were not identified with the search strategy mentioned above. Investigation of indexing revealed that the majority of articles on PDAs are assigned to the subject headings "Microcomputers" and "Computer peripherals." As the National Library of Medicine does not yet have a subject heading for PDAs, indexing into medical subject headings is not uniform. This fact may explain the perceived lack of information about handheld computing. Other search strategies included the review of reference lists and bibliographies of published work as well as internetbased reports found by using standard internet search engines. Our search generated over 119 relevant articles, about half of which provide information relevant to medical applications of handheld devices. To provide a widespread overview of this topic, we included subjective as well as objective descriptions. Despite our efforts to generate a comprehensive literature database, we subsequently discovered various other relevant articles, supporting our concerns about indexing this topic.

Although this review aims to summarize objectively the current literature, numerous software and hardware products are available that have not been studied or discussed in the medical literature. To be as comprehensive as possible we have listed many of these applications in Table 1, but obviously new products are emerging on a daily basis. The reader is referred to the Medical Handheld Software Collections and University Medical Handheld resources (Table 1) for up-to-date product lists.

\section{Personal Digital Assistants in Medicine}

A frequently asked question is which of the increasing number of PDAs should be used. Various medical and lay publications review benefits and disadvantages of the different hardware platforms and operating systems. ${ }^{4}$ Functionality is the first important question that needs to be addressed before purchas- ing a handheld device. If the PDA is meant to replace a paper planner, the memory requirements are significantly less than if it is to be used as a clinical reference. ${ }^{4}$ Memory is an important point to consider because many medical resources require a large amount of memory. The recent introduction of external memory sources (e.g., memory card, memory stick) has added to the diversity of choice.

Several different types of PDA are available. They can be divided into two main groups according to their operating system: Palm (Palm Inc., Santa Clara, CA) or Windows CE, now called Pocket PC (Microsoft, Seattle, WA). Of these, the Palm operating system (Palm OS) is currently more popular. ${ }^{5,6}$ A brief comparison of the two operating systems can be found in Table 2. More medical software applications are available currently for the Palm OS than for any other handheld operating system. ${ }^{5}$ The amount of medical software for the Windows CE operating system is small but growing rapidly. ${ }^{7}$ PDAs manufactured by Palm, Handspring Handera, Sony, and IBM, among others, use the Palm OS. Microsoft's Pocket PC and Handheld PC are used on Hewlett-Packard, Compaq, Sanyo, Toshiba, and Casio devices. The Windows platform offers wider functionality, with better screen resolution and larger memory, but may be more difficult to master. The two operating systems do not interface easily. ${ }^{4}$ Thus, when a PDA is purchased, the following points need to be taken into consideration: what platform colleagues are using, the type of platform interfacing best with your healthcare informatics system, the intended role of the handheld device, and personal preferences. ${ }^{4,5}$

\section{Basic Functions}

The basic functions of PDAs can be quite valuable in medical practice. These functions include an address book, a scheduler, a to-do list, and a memo function. For example, a useful aspect is a scheduler alarm system, which can be used as a reminder for appointments or meetings. To-do lists, schedules and address books can be categorized to organize and synchronize departmental tasks. ${ }^{8,9}$ Computerized dissemination of synchronized schedules, telephone and pager lists, and resident training manuals can also be installed onto the handheld devices. ${ }^{10}$ The small touch-screen keyboard or handwriting recognition system used for data entry is a barrier to the efficient usage of handheld devices for many people. Wright et al. showed that adults over 55 years are significantly less accurate and slower when entering text via the touch-screen. ${ }^{11}$ Even after additional practice 


\section{Table 1}

A List of Websites Related to Medical Use of Handheld Computers (as of June 2002)

\begin{tabular}{|c|c|}
\hline Application & Comment \\
\hline \multicolumn{2}{|l|}{ Medical handheld software collections } \\
\hline HealthyPalm Pilot (www.healthypalmpilot.com) & A well-organized collection of medical shareware \\
\hline Handango (www.handango.com) & Collection of medical and non-medical software \\
\hline PalmGear H.Q. (www.palmgear.com) & Large variety of medical and non-medical software \\
\hline Memo Ware (www.memoware.com) & Database of medical and non-medical references \\
\hline \multicolumn{2}{|l|}{ Handheld medical publishers } \\
\hline Franklin Electronic publishers (www.franklin.com) & $\begin{array}{l}\text { Electronic book collection for various handheld operating systems } \\
\text { from a variety of publishers }\end{array}$ \\
\hline Skyscape (www.skyscape.com) & Wide range of proprietary and other medical books \\
\hline Handheldmed (www.handheldmed.com) & Collection of medical books for Palm and Windows \\
\hline \multicolumn{2}{|l|}{ University medical handheld resources } \\
\hline $\begin{array}{l}\text { Technology Application Unit, Mount Sinai Hospital, Toronto } \\
\text { (www.medtau.org) }\end{array}$ & Handheld computer medical research and links \\
\hline $\begin{array}{l}\text { University of Connecticut Healthcare Center } \\
\text { (http:/ / library.uchc.edu/pda/) }\end{array}$ & Numerous links to medical PDA information and sites \\
\hline Dalhousie University (http:/ / www.medicine.dal.ca/palm/) & Handheld review and numerous links \\
\hline $\begin{array}{l}\text { Arizona Health Sciences Library } \\
\text { (http://educ.ahsl.arizona.edu/pda/index.htm) }\end{array}$ & Reviews, bibliography and links \\
\hline QUAIL (http:/ / www.portablehealth.com/quail/) & Research into handhelds for palliative care and anesthesia \\
\hline SUNY Downstate (http:/ / ect.downstate.edu/support/palm/) & Reviews of medical handheld applications \\
\hline \multicolumn{2}{|l|}{ Document readers } \\
\hline AportisDocMobile (www.aportis.com) & Document reader for commonly used "doc" format files \\
\hline TealDoc (www.tealpoint.com) & Document reader for multiple document formats \\
\hline Documents to Go (www.dataviz.com) & Allows viewing of Microsoft word processing and spreadsheets files \\
\hline isilo (www.isilo.com) & Document/HTML reader, with free document converter \\
\hline Wordsmith (www.bluenomad.com) & Document editor and reader \\
\hline QED (www.qland.de) & Document editor and reader \\
\hline Acrobat reader (www.adobe.com) & PDA reader and converter for Acrobat files \\
\hline
\end{tabular}

Access to medical literature

Avantgo (www.avantgo.com)

HealthProLink (www.healthprolink.com)

Ovid at hand (ww.ovid.com)

JournalToGo (www.journaltogo.com)

\section{Pharmacopoeias}

Epocrates (www.epocrates.com)

ABX Guide (http: / / hopkins-abxguide.org/)

Lexidrug (www.lexi.com)

Mobile Micromedex (www.micromedex.com)

A2Zdrugs, DrDrug (www.skyscape.com)

Physicians' Desk Reference (www.pdr.net)

AHFS Drug information (www.ashp.org/ahfs)

\section{Patient tracking}

Patient tracker (www.handheldmed.com)

WardWatch (www.torlesse.com)

Patient Keeper (www.patientkeeper.com)

Pocket Chart (www.gemedicalsystems.com)

Digital-Doc (www.digital-doc.com)

\section{Specialty specific}

Framingham calculator (www.statcoder.com)

Medicalcalculator (www.netexperience.org)

Med Calc (www.medcalc.be)

Med Math (www.palmgear.com)

Free program to capture webpages and healthcare publications Access to pharmaceutical information

Literature searches on a handheld device

Access to downloadable article summaries

Free drug reference database

Free drug database developed by Johns Hopkins University

Customized extensive drug database

Customized extensive drug database

Customized drug databases

2002 Physicians' Desk Reference

American Hospital Formulary Service

Mobile patient record for Palm OS and Windows CE

Palm-based patient record

Electronic medical record for Palm and Windows

Electronic medical record for Windows CE

Electronic medical record for Pocket PC

Tool for risk estimation of specific diseases

Medical calculator

Medical calculator

Medical formulae 
A List of Websites Related to Medical Use of Handheld Computers (as of June 2002) (continued)

\begin{tabular}{|c|c|}
\hline Application & Comment \\
\hline Med Rules (pbrain.hypermart.net) & Medical diagnosis tools \\
\hline PregPro, (www.thenar.com/pregcalc) & Calculator for pregnancy related problems \\
\hline Pediatric critical care (www.mverive.com) & Access tool for evidence-based information \\
\hline ICD 9-Codes (www.aafp.org) & ICD 9-Codes \\
\hline Risk calculator for Hyperlipidemia (www.nhlbi.nih.gov) & Tool for risk calculation \\
\hline \multicolumn{2}{|l|}{ Other handheld medical sites } \\
\hline Ectopic Brain http:/ / pbrain.hypermart.net/ & Palm-related information and links \\
\hline RN Palm (http://www.rnpalm.com/) & Excellent resource for handhelds for nursing \\
\hline Dr.Cooper.palm (barmacooper.com/drcooper.palm/enframes.html) & All about Palms for medicine and multinational links \\
\hline
\end{tabular}

with the touch-screen, many entries still contain errors. Recent advances in this field include the availability of portable keyboards and thumb keyboards. Suzewits gives a detailed description of how to download and install files from the internet. ${ }^{12}$ Many websites provide access to downloadable medical software applications. Several medical publications list these websites, but often the internet addresses are outdated by the time of publication. Willyard established a list with several popular medical and general-purpose Palm-oriented websites and software archives. ${ }^{13}$ Document readers are important applications that come packaged with some devices or as additional software. These enable users to view text files such as books, medical references and documents downloaded from the internet. Several vendors offer reader programs, including AportisDoc Mobile (www. aportis.com) and TealDoc (www.tealpoint.com). ${ }^{13}$ AportisDoc provides a solution for storing documents of unlimited size on the handheld device. Ebell and Willyard review several readers, 3,13 but even these relatively recent publications are outdated, omitting popular products such as iSilo (www.isilo.com). iSilo allows the user to easily convert common word processor documents into Palm format for installation onto the handheld. Table 1 lists several other document readers and provides several helpful websites, accurate as of June 1, 2002.

\section{Access to Medical Literature}

Access to information at the point of care can be extremely valuable in view of the growing burden of new medical information available, the increased expectations to follow guidelines, formulary restrictions, and the time limitations placed on physicians. ${ }^{5}$ One of the earliest formal studies used the Newton PDA (Apple Computers, Cupertino, CA) in a residen- cy training program, for reference information access. ${ }^{2}$ These devices clearly met an unfilled need, but the importance of providing comprehensive information was emphasized. Various software applications allow access to internet-based literature. Avantgo (www. avantgo.com) can be used to capture web documents and health care publications to be displayed on a PDA. ${ }^{6,8}$ Avantgo is a free software program that can be installed onto the handheld device and allows the user to download website information on a daily basis. Journal websites can be connected to the device and are updated with each synchronization. Thus, abstracts as well as full articles in relevant medical journals can be accessed on the handheld computer.

Most sites are meant for viewing on a fullsize computer screen and are therefore poorly formatted on a PDA. However, many sites now offer a PDA-optimized version of certain pages. ${ }^{6,8}$ HealthProLink (www.healthcareprolink.com) is a tool to obtain selected medical journal articles. ${ }^{14} \mathrm{~A}$ wireless internet connection on the PDA helps to access the information directly from the website. ${ }^{8}$ OVID is one of the main search engines for medical literature used by physicians. With Ovid@Hand (www.ovid.com), searches can be ordered on the handheld device. Through a synchronization, abstracts of journal articles can be retrieved and reviewed. This application gives instant access to vital information about drugs, drug interactions, and evidence-based topics-all with the convenience of handheld delivery.

Many textbooks and handbooks have recently become available in handheld format for both Palm OS and Windows CE/Pocket PC. They are available from Franklin Electronic publishers (www.franklin. com), Skyscape (www.skyscape.com), and other publishers (Table 1). 
Comparison of Windows CE and Palm Operation System (Palm OS)

\begin{tabular}{ll}
\hline WINDOWS CE & PALM OS \\
\hline $\begin{array}{l}\text { General } \\
\begin{array}{l}\text { Different versions required for different central processing units } \\
\text { and different vendors }\end{array}\end{array}$ & $\begin{array}{l}\text { Optimized for specific hardware } \\
\text { Only one version, with little variation in hardware } \\
\text { Used only for the specific Palm platform but highly customized for } \\
\text { this function } \\
\text { but not customized for any }\end{array}$ \\
\end{tabular}

\section{Hardware}

Compatible with different screens, keyboards, modems, peripheral devices, etc.

\section{Functions}

Can be used for ultra-light notebook PC, Windows terminals, computers for cars

\section{Programs}

Designed to run multiple tasks and programs simultaneously

\section{Software development}

More complex, fewer third party applications developed

\section{Usability}

More complex, based on standard Windows interface translated to a small screen

\section{Devices}

HP, Compaq, Sanyo Toshiba, Casio, other

\section{Pharmacopoeias}

Drug information databases, or pharmacopoeias, are ideally suited for formatting onto a handheld device. An example of rapid dissemination is ePocrates (www.epocrates.com), which has quickly become one of the most popular handheld drug databases. According to the ePocrates company, as of March 2002, more than 500,000 users had downloaded the program since its introduction in November 1999. This free medical reference guide for Palm OS devices provides a comprehensive drug list, adult and pediatric dosing guidelines, drug interaction guidelines, retail prices of drugs, and common side effects. ${ }^{15}$ Physicians reported that ePocrates saves time during information retrieval, is easily incorporated into their usual workflow, and improves drugrelated decision making. ${ }^{16}$ Greiver, a Canadian physician, finds that ePocrates is a helpful tool for family physicians to enter formulary code numbers for medications that are restricted by drug benefit programs. ${ }^{17}$ Updates can be automatically downloaded and installed, a significant advantage over older methods of keeping references up to date. ${ }^{18}$ In January 2001 ePocrates published qID, an infectious disease application designed specifically for hand-
Many parts cannot be changed such as screen, controller chips, etc

Function limited to PDAs and PDA-telephone combinations

Designed to run limited number of tasks and programs, does not multitask

Technically less demanding, resulting in many products produced by independent developers

Simpler to use, developed for a small screen

Palm, Handspring, Handera, Sony, IBM, other

held devices that rapidly attained the same number of users as ePocrates. ${ }^{19}$ These databases are available for free downloading from the ePocrates website, however users must register with ePocrates, Inc. and submit basic demographic and practice information. This information, and even information about drugs reviewed, may be sold by ePocrates to the pharmaceutical industry. ${ }^{20}$ This database is currently not available for Windows CE.

The ABX Guide (http://hopkins-abxguide.org) is a free database developed at the Division of Infectious Disease at Johns Hopkins University. Antibiotic information can be searched by organ, pathogen, or drug for diagnostic and therapeutic information. This database is regularly updated by experts, ${ }^{5}$ and transferred to the Palm during a synchronization process.

Many different customized drug databases are available. Embi provides a list with general drug references, antimicrobial drug references and general medical references, including brief descriptions about the database and its cost. ${ }^{5}$ References to further pharmacopoeias and a brief description can be found in Table 1. An evaluation of different pharmacopoeias in the setting of an intensive care unit compared sev- 
eral handheld pharmacopoeias in a clinical environment. Handheld drug references were found to have the potential to meet point-of-need requirements in critical care, but significant differences exist in available products in terms of content and ease of use. ${ }^{21}$

\section{Patient-tracking Applications}

Patient data management and sign-over between physicians is an area in which the risk of errors is high and in which PDAs may play a significant role. Documents containing patient information can be transferred from the computer desktop onto a handheld device or created directly on the handheld device. The data and changes that are made to the database can be transferred to the PDA with subsequent synchronizations. ${ }^{9}$ Infrared beaming allows transfer of data between PDA users. A study by Lapinsky et al. using focus group analysis of PDA users found that patient-care data management using a handheld device was not considered valuable, unless it is integrated into the hospital computer system. ${ }^{22}$ However, the long-term goal of integrating e-prescribing, physicians' notes, radiology, and laboratory handheld capacities for physicians in outpatient clinics as well as in hospitals will dramatically improve functionality. ${ }^{23}$

Handheld devices have been used to monitor patients' health and to keep medical staff informed about the patient's condition. Many customized patient-tracking programs are available. Patient Tracker (www.handheldmed.com) gives the user the option to enter patient records, including demographics, laboratory results, medication/allergy lists, test results, and radiology reports. It is available for Windows $C E$ as well as for Palm OS. WardWatch (www.torlesse.com) was designed specifically for Palm organizer to aid medical staff involved in ward rounds. It is intended to be used for recording investigations, medications, dosages, and consultations. Medical PocketChart (www.gemedicalsystems.com) is an electronic medical record keeper for Windows CE.

The purpose of a handheld implementation program published by Szynal was to optimize the admission condition of candidates for transplant surgery. ${ }^{24}$ Before the handheld program was applied, some patients suffered serious consequences when their deteriorating health was reported too late. A series of questions, developed by the university medical staff, was programmed for the handheld device. Information entered daily by the patient is electronically sent to the university on a regular basis, using a built-in computer modem. This program was found to be highly beneficial.
Shiffman et al. found in a trial with randomly selected pediatricians that handheld computers providing guideline-based decision support are associated with increased adherence to guideline recommendations. However, there were no significant differences in immediate disposition or subsequent emergency department visits. ${ }^{25}$

Sophisticated handheld systems have been developed by the group at Cedars-Sinai Medical Center in Los Angeles. A clinical alert system identifies serious or life-threatening clinical events, using a configurable, rule-based analysis of a clinical information system. ${ }^{26}$ Alerts are transmitted wirelessly to the clinician's PDA. No outcome evaluation of this system has been performed. More recent developments include PDA-based wireless access to a clinical data repository, using the Palm VII wireless device. ${ }^{27}$ This method allows searchable access to select clinical data. Although effective, this system was limited by bandwidth and resolution issues, which will be addressed by improving technology.

\section{Medical Education}

Electronic data collection is becoming increasingly important to manage information in the medical education environment. Handheld devices have been used for the evaluation of training for medical students, to help monitor students' clinical experience, and to ensure that students are benefiting from the provided teaching. ${ }^{28}$ Because a large amount of information needs to be collected, many institutions use an electronic method for data collection. ${ }^{28,29}$ Patient contacts can be entered onto a handheld computer spreadsheet by the students, sorted automatically, and evaluated easily. ${ }^{28,29}$ Handheld computers have been found to be a practical and feasible way to identify gaps in the student's education and experience so that action can be taken to address them. Because it is possible for the students to take the handheld computer into settings where they come into contact with patients, reporting of contacts is maximized. ${ }^{28}$ Sullivan et al. describe a project in which handheld devices were integrated into a family medicine clerkship to document patient contact and educational experiences. ${ }^{30}$ Preliminary results suggested that this effort is an effective way to use current technology to enhance student learning and program evaluation. Another advantage is that additional software can be added, providing reference material that can be accessed during clinical training. ${ }^{29}$ Because significant resistance still limits the implementation of mobile technology among caregivers, an early intro- 
duction may be an important factor in preparing future physicians for technologic innovations. ${ }^{29}$

In a survey sent to the program directors of all American Academy of Family Physicians and American College of Osteopathic Family Practice residency programs in the United States, Chriswell et al. found that of the $50 \%$ who responded, two-thirds were using handheld devices in their residency programs. An additional $14 \%$ had plans to implement PDAs within the following 24 months. ${ }^{31}$

A handheld computer procedure log for tracking residents' procedural experience is an important tool to ensure an adequate educational experience..$^{32} \mathrm{~A}$ number of studies describe procedure logging in different areas of medicine with handheld devices. Garvin et al. evaluated the use of handheld devices in family medicine for procedure documentation. Implied in their article was an overall cost saving to the program due to increased efficiency. ${ }^{33}$ Bird and coworkers found that the use of a handheld device was associated with an increase in first-year emergency medicine residents' documentation in three of 20 procedures. ${ }^{34}$ The mean number of procedures, encounters and followups performed per resident using a handheld device was compared with a historical control group. It was concluded that the overall time savings in using a resident procedure database may result in a transition to a handheld computer-based procedure log. Logged procedures can be sent to a central database and analyzed for residency management. ${ }^{32,35}$ Nicolaou et al. report that a handheld procedure log has achieved high reliability and data integrity, low data entry workload and rapid feedback for residents and the program director concerning the residents' procedural experience. ${ }^{36}$ This approach was associated with very low cost, training overhead or complexity. ${ }^{36}$

PDAs have been used to create the required Residency Review Committee reports for training in obstetrics/gynecology. Data collection and transfer processes using a handheld computer system synchronized to a central database were shown to be faster for residents and program directors than paper-based systems. ${ }^{10}$

A logbook is an essential component of the assessment of anesthesia trainees in the United Kingdom. It was shown that this logbook can be replaced by a handheld device for data collection. ${ }^{37}$

Handheld devices are described to evaluate teaching interactions. A clinical teaching evaluation form was transferred into a PDA format. Preliminary feedback indicates that entering evaluation data has prompted participants to improve and change their teaching profile. ${ }^{38}$ A randomized controlled trial demonstrated comparability of the hand-held computer method to the paper-and-pencil method in obtaining survey information. ${ }^{39}$ Handheld devices also have a role in the settings of objective structured clinical examinations (OSCE). Written checklists are replaced by programs installed on handheld devices. The given tasks and their solutions can be accessed by the observer by opening drop-down lists on the handheld screen. At the end of the examination, the data of all participating candidates are synchronized and transferred to a database on a desktop computer, and immediate, formative feedback can be elaborated. ${ }^{40}$ This system allows rapid statistical evaluation of examination results.

\section{Research}

Handheld computers with customized software have been used for research data collection. Shiffman et al. describe the use of PDAs for the evaluation of craving and withdrawal symptoms of patients in a smoking cessation program. ${ }^{41}$ Rather than asking patients to recall their experiences retrospectively, the patients were able to enter information at the precise moment in their daily life in their natural environment. The patient can be reminded to enter information by an audible beep from the device. Handheld devices have also been used successfully to collect sociodemographic characteristics of tobacco use in Bombay. ${ }^{42}$ Bourne et al. found electronic point-of-care data collection appropriate, particularly in high-volume, highcost surgical interventions such as a total joint replacement. ${ }^{43}$ Caro et al. compared the accuracy of electronic data collection with a paper questionnaire. ${ }^{44}$ They found that successful implementation depends on proper training of the respondent in the handling of the electronic instrument. Lal et al. found that the total time for the data collection on a handheld device and downloading to a personal computer was $23 \%$ faster than hand recording and generated 58\% fewer errors. ${ }^{45}$ Pendragon Forms (www.pendragonsoftware.com) is one example of a handheld application that allows the development of a customized database for viewing, managing and exporting data. It provides a fast and easy way to create a data collection system for the Palm computing platform. The collected data are transferred during each synchronization into a desktop Access database for subsequent analysis.

\section{Business Management}

It may be difficult for medical staff and assistants to communicate regarding patient care, meetings, and 
call schedules. PDAs have an important role in improving business efficiency by improving coordination of physicians' schedules, making billing more time- and cost-effective, and improving physicians' communication. ${ }^{46}$ Software for coding and billing is improving and becoming more commonplace. ${ }^{5}$ Billing information can be entered by the physician during rounds in the hospital, transferred to a central database on synchronization, and viewed by business office staff. A program within the PDA instantly checks charges for accuracy and completeness and prompts the provider to make the necessary corrections. ${ }^{47}$ This approach may provide quicker billing and improve cash flow. ${ }^{48}$ It was shown that upfront costs, including evaluation, database configuration, implementation, training, software, and handheld hardware, still demonstrate return of investment rapidly after implementation of a PDA..$^{48}$

\section{Prescribing / Pharmacy}

PDAs integrated into the Department of Pharmacy have been shown to improve access to patient and drug information by medical staff and to decrease medication error rates. ${ }^{49}$ Brody et al. implemented and evaluated the use of PDAs to document clinical interventions by pharmacy residents. ${ }^{14}$ Scientific calculators, drug information databases, and pharmacokinetic programs were rated as highly useful. Using PDAs for reporting clinical interventions was useful but did not save time compared with paper. It was suggested that funding for computer program upgrades should be included in proposals for similar projects. $^{14}$

Patients' medication profiles, generated by the pharmacy department and updated daily, and patients' discharge summaries can be formatted for PDAs. Through a synchronization between the desktop and the handheld devices, PDA users are able to obtain current medication profiles and can access all previous discharge summaries. The mobile pharmacist has immediate electronic access to patient and drug information. On the discharge day, the current medication list and dosages can be directly sent to a printer via an infrared port. ${ }^{49,50}$ Grasso states that this strategy may prevent transcription errors that occur when the process is done by hand. ${ }^{99}$ However, at the time of publication of this article the collection of outcome data about the impact of the devices on discharge medication transcription error rates was not completed.

Because critically ill patients are at increased risk for drug-related problems, the pharmacist in the inten- sive care unit (ICU) serves as a resource for other health care providers. Integrating PDAs into patient care can simplify patient data management and overcome some of the limitations of the paper format, such as the inability to automatically sort and analyze data. ${ }^{51}$ Data are generally clearer and better organized compared with data on paper forms.

Electronic prescribing via handheld computers has also become an increasingly popular tool. Thousands of physicians are now writing prescriptions electronically and transmitting them to the pharmacy without manual intervention. ${ }^{15}$ This approach may reduce errors caused by illegible handwriting and transcription. Parker et al. developed a program for a handheld device that enables the physician to prescribe medications according to specific health plan formularies. ${ }^{50}$ This method markedly reduces pharmacy call-backs and saves time for the physician and staff. Despite the challenge of integrating an e-prescribing system into the hospital setting, the sooner hospitals realize the potential benefits of handheld devices, the more incentive developers will have to improve technology. ${ }^{23}$

\section{Specialty-specific Applications}

\section{Family Medicine}

Handheld computer technology may provide general practitioners with a useful tool for practice management and at the same time deliver optimal patient care for the diverse clinical problems that they encounter. Criswell suggests that handheld computers seem to be widely accepted in family practice residencies and are currently used for the roles described above. ${ }^{31}$ At present, the most active users of handheld devices are doctors in small group practices outside hospitals. ${ }^{23} \mathrm{~A}$ handheld device is a helpful tool in family practice to access evidence-based information at the patient's bedside. ${ }^{17}$ The Framingham calculator (www.statcoder.com) estimates patients' risk of cardiac events over the next 10 years and gives family physicians the opportunity to calculate how much the risk decreases with the cessation of smoking. ${ }^{17}$ A medical calculator (www.medcalc.be) allows the physician to determine the patient's body mass, predicted peak flow or other clinical parameters. A pregnancy calculator (PregPro: www. thenar.com) stores the patient's last menstrual period, calculates weight gain, fetal weight, and biparietal parameters and reminds the user to order lab tests at appropriate times. Many of these medical calculations have been consolidated into applications such as MedRules (http:/ / pbrain.hypermart.net) or MedCalc (www.palmgear.com). 
A handheld device is ideally suited for rapid access to diagnostic codes. Many PDA-oriented websites offer downloadable complete ICD-9 lists as databases. However, for the most part, they are cumbersome and take up a large amount of memory. Family Practice Management publishes a list with ICD-9 codes that are most often used in family practice. ${ }^{13}$

\section{Pediatrics}

Weigle at al. focus on web resources for the PDA to access pediatric information. ${ }^{6}$ PedsCCM offers an Avantgo-optimized version of its Evidence-Based Medicine Journal Club. ${ }^{6}$ The Evidence-Based Journal Club offers peer-reviewed, structured critical appraisals of timely clinical research studies in critical care medicine. PICU Tools is a collection of calculators and reference guides for pediatric critical care. ${ }^{6}$

Clinical practice guidelines that are developed using evidence-based methods encapsulate the current understanding of best clinical practice. Finding effective strategies to implement these guideline recommendations is an area of active investigation. Shiffman et al. showed in a randomized, prospective trial with private-practice pediatricians that handheld computers can be effective in improving physicians' adherence to a clinical practice guideline. ${ }^{52}$ Most of the reported dissatisfaction was related to deficiencies in the user interface. The most serious complaint was related to the excessive time required to use the system in a clinical setting. Carroll et al. describe the design, software and hardware selection, and preliminary testing of a PDA-based patient data and charting system in the setting of a neonatal intensive care unit..$^{53}$ This system will be the subject of a subsequent study to determine its impact on patient outcomes and clinician efficiency.

\section{Pain Management}

Handheld devices are a popular tool for patient data collection and transfer. They have been used for patients with chronic pain, because they allow physicians to assess and document pain at the point of the patient's complaint. ${ }^{54}$ Data about the patient's previous therapeutic regimens can be assessed at the bedside. Since the small handheld computer can be carried in the brief case or pocket, data entry can take place at any time. A number of studies have evaluated the use of handheld devices in pain management and palliative care. At present most of these studies have been submitted for publication or presented in poster form. ${ }^{55}$

\section{Critical Care}

A qualitative study of handheld devices in an academic intensive care unit demonstrated that they are convenient and functional. ${ }^{56}$ The most valuable role was found to be access to medical reference information and drug dosing. Training in the use of handheld devices was identified as an area of possible improvement. Customized software and hardware would improve the value of these devices. In a small crossover study, a critical care handbook and its electronic equivalent loaded on a handheld were compared in an ICU setting. No advantages of either format were noted, but the handheld device had the potential to carry five times the information in a smaller format. ${ }^{56}$

\section{Cardiology}

To decrease the time to reperfusion therapy for patients with acute myocardial infarction, the interpretation of prehospital EKGs is important. Computer-based applications and wireless technology have allowed the transmission of 12-lead EKG waveforms from remote locations to hospitals. A study performed by Pettis et al. proved that handheld devices can be used safely for the interpretation of EKGs. ${ }^{57}$ It showed that cardiologists make the same diagnosis when viewing EKGs on a handheld device as they do when viewing paper displayed EKGs.

In May 2001, the National Cholesterol Education Program released a new set of guidelines for lipid testing and treatment. Because calculating the risk for an individual patient requires the physician to go through a number of calculations, the National Heart, Lung and Blood Institute published a free program downloadable onto a handheld device (wwww.nhlbi.nih.gov).

\section{Handheld Systems: Implementation Issues}

\section{Patient Confidentiality}

Patient confidentiality during use of handheld devices is an area of significant importance and potential concern. ${ }^{58}$ Password protection ${ }^{9,28,49}$ is the most commonly used method to protect patient confidentiality. A coded patient identifier ${ }^{28}$ or the last digits of the social security number ${ }^{10}$ can be used instead of the patient's name. Although handheld devices have a locking security feature, users must remember to activate it each time that they want to secure their computer. Alternatives include Sign-On (www.cic.com) or TealLock (www.tealpoint.com). Both programs enable users to program palm-tops to 
lock-out automatically after a user-defined period of inactivity. These programs offer the ability to use signature, initial, on-screen buttons or a number pad to unlock the computer. ${ }^{59}$

\section{Costs}

An important factor that may affect the integration of handheld devices into the medical setting is cost. Costs, which tend to vary depending on the package, include the software, the server, upgrades to the hospital's network, the handheld units themselves, and support. ${ }^{15}$ One may assume that cost return will occur through decreasing charting time, fewer errors, and more time left for patient care, but so far a comparison of costs before and after integration of mobile technology has not been done. ${ }^{15}$ Other barriers include a lack of standards and limited bandwidth for data transfer. ${ }^{15}$ Nevertheless, the costs of a handheld device are significantly lower per unit than desktop or laptop computers.

\section{Discussion}

Handheld computers have significant potential to improve medical practice beyond serving as an address-book or calendar. The increasing implementation of this technology is impressive, but handheld devices have not yet become a standard medical tool. There are three components for successful system implementation: the technology, the software, and the people who need to use it. If any of these three points is not optimized, the system will be established but not used. ${ }^{60}$ Although hardware and software are available, they may not be optimized for the medical environment. Furthermore, the consumer's interest may be the limiting factor to a successful system implementation. Large studies are planned to evaluate the effectiveness of PDA programs for improving patient care efficiency and reducing medical error. ${ }^{61}$

A comprehensive literature search identified a relatively small number of articles providing evidencebased information about the use of handheld devices in medicine. Most publications consist of reports of clinical experience with the use of PDAs or innovative uses without significant substantiating data. We hope that this review serves to increase awareness among physicians about the current and possible uses for handheld devices in various fields of medicine. We also hope to provide ideas for further projects. Although this technology appears attractive, further studies are necessary to prove benefits in terms of costs, patient outcome, and clinician satisfaction.

\section{Conclusion}

Handheld devices offer increasing support to physicians in their daily clinical activity and an increasing potential for future use. A growing body of literature supports the use of handheld devices in a variety of medical settings. With the rapid advances in this technology, the mobile computer may well become an essential medical tool.

\section{References}

1. Fisher J, Wang R. The Cure Is in Hand. WR Hambrecht and Co, 2000.

2. Labkoff SE, Shah S, Bormel J, Yee Y, Greenes RA. The Constellation project: experience and evaluation of personal digital assistants in the clinical environment. Proc Annu Symp Comput Appl Med Care 1995;678-682.

3. Ebell MH, Gaspar DL, Khurana S. Family physicians' preference for computerized decision-support hardware and software. J Fam Pract 1997;45(2):127-128.

4. Schneider S, Kostecke R, Tokazewski J. Buying your first PDA. Fam Pract Manage 2001;8(7):50-51.

5. Embi PJ. Information at hand: using handheld computers in medicine. Cleve Clin J Med. 2001;68(10):840-842, 845-846, 848-849.

6. Weigle CG, Markovitz BP, Pon S. The Internet, the electronic medical record, the pediatric care unit, and everything. Crit Care Med. 2001;29 (8):N166-N176.

7. Ebell M, Rovner D. Information in the palm of your hand. J Fam Pract 2000;49(3):243-251.

8. Chyna JT. Making the most of you PDA (personal digital assistant). Healthcare Exec 2001;16(3):66.

9. Luo J, Hales R, Hilty D, Brennan C. Clinical computing: Electronic sign-out using a personal digital assistant. Psychiatr Serv 2001;52(2):173-174.

10. Malan TK, Haffner WH, Armstrong AY, Satin AJ. Hand-held computer operating system program for collection of resident experience data. Obstet Gynecol 2000;96(5 Pt 1):792-794.

11. Wright P., Bartram C, Rogers N, et al. Text entry on handheld computers by older users. Ergonomics 2000;43:702-716

12. Suzewits J. Downloading applications for your PDA. Fam Pract Manage 2001;8(9):53-54.

13. Willyard KE. A palm-top computer in every practice? Fam Pract Manage 2000;7(8):59-60.

14. Brody JA, Camamo JM, Maloney ME. Implementing a personal digital assistant to document clinical interventions by pharmacy residents. Am J Health-Syst Pharm 2001;58(16): 1520-1522.

15. Kelly, J. Going wireless. Hosp Health Netw 2001;74(11): 65-66,68.

16. Rothschild JM, Lee TH, Bae T, Bates DW. Clinician use of a palmtop drug reference guide. J Am Med Inform Assoc 2002;9(3):223-229.

17. Greiver, M. Evidence-based medicine at the palm of your hand. Can Med Assoc J. 2001;164(2):250.

18. Hogan R. New media: Therapeutics. JAMA 2001:286(2): 229-230.

19. Shalo S. Bugs, drugs and the success of viral marketing. Pharmaceut Exec 2001. 21(5):100-102.

20. Johnson MT. New media: Infectious disease. JAMA 2001:286(2):230-232. 
21. Lapinsky SE, Weshler J, Burry L, et al. Evaluation of a handheld electronic pharmacopoiea in the intensive care unit. Am J Respir Crit Care Med 2002;165(8):A105.

22. Lapinsky SE, Weshler J, Mehta S, Varkul M, et al. Handheld computers in critical care. Crit Care 2001;5(4):227-231.

23. Stammer L. A show of handhelds. Healthcare Inform 2001;18(4):37-40, 42,44

24. Szynal D. PDAs come in handy. Health Data Manage 2001;9(8):18-20.

25. Shiffman RN, Freudigman KA, Brandt CA, Liaw Y, Navedo DD. A guideline implementation system using handheld computers for office management of asthma: Effects on adherence and patient outcomes. Pediatics 2000;105(4):767-773.

26. Shabot MM, LoBue M. Real-time wireless decision support alerts on a Palmtop PDA. Proc Annu Symp Comput Appl Med Care. 1995; 174-177

27. Duncan RG, Shabot MM. Secure remote access to a clinical data repository using a wireless personal digital assistant (PDA). Proc AMIA Symp. 2000;210-214

28. Alderson TS, Oswald NT. Clinical experience of medical students in primary care: Use of an electronic log in monitoring experience and in guiding education in the Cambridge Community Based Clinical Course. Med Educ 1999;33(6): 429-433.

29. Bass SG. Wireless computing. Medical student and mobile medicine. MD Comput 2000;17(6):27.

30. Sullivan L, Halbach JL, Shu T. Using personal digital assistants in a family medicine clerkship. Acad Med 2001;76(5):534-535.

31. Chriswell DF, Parchman ML. Handheld computer use in U.S. Family Practice Residency Programs. J Am Med Inform Assoc 2002;9:80-86.

32. Larson JL, Look R, Schiffman B. A hand-held computer-based procedure log. Acad Emerg Med 2001;8(5):583.

33. Garvin R, Otto F, McRae D. Using handheld computers to document family practice resident procedure experience. Fam Med 2000;32(2):115-118.

34. Bird SB, Zarum RS, Ranzi FP. Emergency medicine resident patient care documentation using a hand-held computerized device. Acad Emerg Med 2001;8(12):1200-1203.

35. Fischer S, Lapinsky SE, Weshler J, et al. Surgical procedure logging with use of a hand-held computer. Can J Surg 2002; 45:345-350.

36. Nicolaou, DD, Davis GL. A distributed asynchronous resident procedure log for hand-held devices. Acad Emerg Med 2001; 8(8):583.

37. Hammond EJ, Sweeney BP. Electronic data collection by trainee anesthetists using palm top computers. Eur J Anaesthesiol 2000;17:91-98.

38. Krippendorf RL, Simpson DE, Schiedermayer D. Promoting reflective teaching with personal digital assistants. Acad Med 1999;74(5):577.

39. McBride JS, Anderson RT, Bahnson JL. Using a hand-held computer to collect data in an orthopedic outpatient clinic. Med Care 1999;37:647-651.

40. Schmidts MB. OSCE logistics-handheld computers replace checklists and provide automated feedback. Objective structured clinical examination. Med Educ 2000;34(11):957-958.

41. Shiffman S, Elash CA, Paton SM, et al. Comparative efficacy of 24-hour and 16-hour transdermal nicotine patches for relief of morning craving. Addiction. 2000;95(8):1185-1195.

42. Gupta PC. Survey of sociodemographic characteristics of tobacco use among 99,598 individuals in Bombay, India using handheld computers. Tobbacco Control 1996;5(2):114-120.

43. Bourne RB, Sibbald WJ, Doig G, et al. Southwestern Ontario Study Group. The Southwestern Ontario Joint Replacement Pilot Project: electronic point-of-care data collection. Southwestern Ontario Study Group. Can J Surg 2001; 44(3):199-202.

44. Caro J J Sr, Caro I, Caro J, Wouters F, Juniper E.F. Does electronic implementation of questionnaires used in asthma alter responses compared to paper implementation? Qual Life Res 2001;10(8):683-691.

45. Lal SO, Smith FW, Davis JP, et al. Palm computer demonstrates a fast and accurate means of burn data collection. J Burn Care Rehabil 2000;21:559-561.

46. Blackman J, Gorman P, Lohensohn R, Kraemer D, Svingen S. The usefulness of handheld computers in a surgical group practice. Proc AMIA Annu Symp 1999:686-690.

47. Monteverdi B. In the palm of your hand. Med Group Manage J 2001;48(8):34-38.

48. Nelson, R. Handhelds capture charges, speed billing cycle. Med Group Manage J. 1999;46(4):18.

49. Grasso BC, Genest R. Clinical computer: Use of a personal digital assistant in reducing medication error rates. Psychiatr Serv 2001;52(7):883-884.

50. Parker GM. Easing workflow in the palm of a physician's hands. Portable POC system saves physician time, patient aggregation. Health Manage Technol 1999;20(10):48-49.

51. Lau, A, Balen RM, Lam R, Malyuk DL. Using a personal digital assistant to document clinical pharmacy services in an intensive care unit. Am J Health-Syst Pharm 2001; 58(13):1129-1132.

52. Shiffman RN, Liaw $Y$, Navedo DD, Freudigman KA. User satisfaction and frustration with a handheld, pen-based guideline implementation system for asthma. Proc AMIA Annu Symp 1999:940-944.

53. Carroll AE, Trczy-Hornoch P, Saluja S. Development of a personal digital assistant (PDA) based client/ server NICU patient data and charting system. Proc AMIA Symp. 2001:100-104.

54. Affleck G, Tennen H, Urrows $S$, et al. Fibromyalgia and women's pursuit of personal goals: A daily process analysis. Health Psychol 1998;17:40-47.

55. Goldstein DH, Van Den Kerkhof EG, Rimmer M. Personal Digital assistants and batch data. A computer model for research and management in acute pain management Services. Poster presented at the American Anesthesiologists' Society Annual Meeting, New Orleans, October, 2001.

56. Lapinsky SE, Weshler J, Varkul M, et al. Handheld computers as a reference source in the ICU. Am J Respir Crit Care Med 2001;163:A258.

57. Pettis KS, Savona MR, Leibrandt PN, et al. Evaluation of the efficacy of hand-held computer screens for cardiologists' interpretation of 12-lead electrocardiograms. Am Heart J 1999;138 (4 Pt 1):765-770.

58. Harris CM. Handheld computers in medicine: The future is not here yet. Cleve Clin J Med 2001;68(10):854-856.

59. Hill DB. How secure is you palm-top? Fam Pract Manage 2001; 8(7):13.

60. Rabinowitz E. Will Palm-size computers make electronic prescribing happen? Manag Care 1999;8(10):56-61.

61. Rosenbloom M, Jaffe W, Adams J. Patient care efficiency and medical error reduction using PDA-based medical information. Acad Emerg Med 2001;8(5):587-588. 